\title{
Transcatheter Occlusion of a Hepatic Vein to left Atrium Fistula: Should we close Venovenous collateral Vessels following Fontan operation?
}

\section{Maya El-Khouri ${ }^{2,5}$, Mariam Arabi ${ }^{2,5}$, Issam El Rassi ${ }^{1,5}$, Ziad Bulbul ${ }^{2,5}$, Fadi Sawaya ${ }^{4}$, Fadi Bitar ${ }^{2,5^{*}}$}

${ }^{1}$ Department of Surgery

${ }^{2}$ Department of Pediatrics and Adolescent Medicine

${ }^{3}$ Department of Radiology

${ }^{4}$ Department of Medicine

5the Children's Heart Centerthe American University of Beirut-Medical Center, Lebanon.

*Corresponding Author: Fadi Bitar, Children's Heart Center, American University of Beirut, Cairo Street, Beirut, Lebanon

Received Date: February 22, 2021; Accepted Date: March 23, 2021; Published Date: March 30, 2021

Citation: Maya El-Khouri, Mariam Arabi, Issam El Rassi, Ziad Bulbul, Fadi Sawaya, Fadi Bitar., (2021) Transcatheter Occlusion of a Hepatic Vein to left Atrium Fistula: Should we close Venovenous collateral Vessels following Fontan operation?. J. Clinical Cardiology and Cardiovascular Interventions, 4(8); Doi:10.31579/2641-0419/148

Copyright: (c) 2021 Fadi Bitar, This is an open-access article distributed under the terms of the Creative Commons Attribution License, which permits unrestricted use, distribution, and reproduction in any medium, provided the original author and source are credited.

\begin{abstract}
Right to left shunt due to abnormal systemic venous drainage to the heart or the pulmonary veins is among the causes of hypoxemia following the Fontan operation. There are conflicting data regarding the closure of the venovenous collateral (VVC) vessels post-Fontan. The embolization of these VVC in older patients may be associated with decreased survival.

We describe a rare fistula draining a right-sided hepatic vein connected to a hepatic venous plexus to a right-sided pulmonary venous atrium in a child with visceral heterotaxy. The patient presented with severe hypoxemia following the Fontan operation. The fistula was successfully occluded by an AMPLATZER ${ }^{\text {TM }}$ Vascular Plug II to resolve the hypoxemia and improve the hemodynamic status.

Approach to venovenous collaterals in older patients with mild cyanosis after the Fontan operation may differ from that in younger children with severe hypoxemia and intrahepatic VVC. Patients with profound cyanosis due to intrahepatic to atrial connection may benefit from the occlusion of these connections. Percutaneous closure of hepatic to atrial connection with significant hypoxemia following appropriate hemodynamic assessment may be beneficial.
\end{abstract}

Keywords: fontan; left atrium to hepatic vein fistula; AVP II; transcatheter closure of fistula

\section{Background}

Fontan procedure is a palliative surgery done as a final stage treatment in patients with single ventricle physiology [1]. This surgery aims to direct blood from both veno cavas directly to the pulmonary arteries [2]. The Fontan procedure can be performed with fenestration, where a residual right to left shunt is left on purpose to decompress the relatively high pulmonary artery pressure or can be done without fenestration [3]. Some schools advocate that fenestration might improve the surgical results in high-risk patients.

Patients with the Fontan circulation are usually slightly desaturated with arterial oxygen saturation in the low 90s, even in the absence of fenestration. In most recent circuit modifications, the coronary sinus drains to the left atrium, causing a modest arterial desaturation of $\pm 2 \%$. Other causes of hypoxemia following the Fontan operation include a residual atrial septum defect or fenestration, intrapulmonary arteriovenous fistulae, and a right to left shunt due to abnormal systemic venous drainage to the heart or the pulmonary veins. Closure of the venovenous collateral (VVC) vessels may be necessary to decrease embolism and hypoxemia. However, Poterucha et al. [4] reported that the embolization of the VVC in older patients after the Fontan operation is associated with decreased survival, as these patients may benefit from the "natural" fenestration that VVCs provide.

Reports of intrahepatic VVC presenting with profound cyanosis following the Fontan operation are rare and usually associated with visceral heterotaxy and a nest of intrahepatic venous plexus. The few reported cases that involved occlusion of these intrahepatic VVC were associated with a favorable outcome [5-10].

We report a rare case of a fistula between a right hepatic vein and a rightsided pulmonary venous atrium in a child with visceral heterotaxy and profound hypoxemia following the Fontan operation. The right-sided hepatic vein was connected to a hepatic venous plexus. The fistula was successfully occluded by an AMPLATZER ${ }^{\text {TM }}$ Vascular Plug II (AVP II) to resolve the hypoxemia and improve the hemodynamic status. The approach of the VVC arising from the hepatic veins maybe be different from the smaller VVC associated with mild desaturation in older children. The decision to occlude the VVCs may depend on their type and location. 


\section{Case presentation}

We report a 7-year old boy, born term to first-degree consanguineous parents, diagnosed with an unbalanced AV canal (single ventricle physiology), abdominal situs ambiguous, severe pulmonary stenosis with confluent pulmonary arteries, and normally related great vessels. The patient underwent Blalock Taussig shunt insertion during infancy, followed by bidirectional Glenn at 18 months.

Cardiac catheterizations before the Fontan operation revealed atrial situs inversus with the pulmonary veins draining into a posterior right-sided atrium (left atrium) and an inferior vena cava (IVC) on the left of the spine draining into the left-sided atrium. There was a tiny connection between the right hepatic vein and the LA.

We performed total cavo-pulmonary circulation without fenestration at the age of 3 years with no significant complications. Immediately post Fontan procedure, the patient had an arterial oxygen saturation of $97 \%$. However, one-month post-procedure, the systemic arterial oxygen saturation declined to $80 \%$ and reached the mid-70s a year later.

Cardiac catheterization post-Fontan revealed a fistula between a right hepatic vein and a right-sided pulmonary venous atrium. The right-sided hepatic vein was connected to the IVC, and a right-sided hepatic venous plexus (Figure 1).

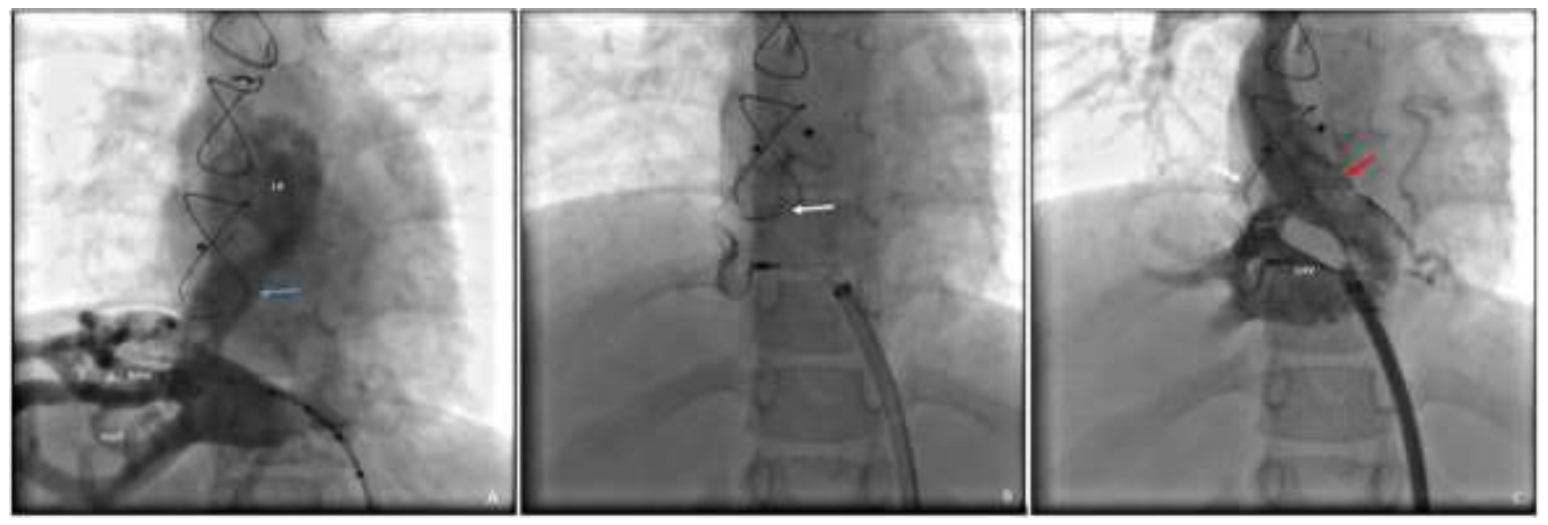

Figure 1: Angiograms in AP projection (A) right hepatic vein $(R V H)$, dividing into multiple sinusoidal channels in the liver (HVP= hepatic venous plexus), and draining into the right sided pulmonary venous atrium (LA) through a fistula (blue arrow) (B) Amplatzer vascular plug type II (white arrow) placed successfully in the fistula, attached to the delivery wire (C) Amplatzer vascular plug type II (white arrow) closing the fistula with no obstruction of the Fontan circulation (red arrow).

Occlusion of the fistula with a $12 \mathrm{~mm}$ TYSHAK® balloon dilatation catheter resulted in a trivial increase in the mean pulmonary pressure from $14 \mathrm{~mm} \mathrm{Hg}$ to $16 \mathrm{~mm} \mathrm{Hg}$ with a rise in the arterial oxygen saturation to the high 90s with appropriate systemic blood pressure. Accordingly, we decided to close the fistula.

A $5 \mathrm{~F}$ Judkins catheter was advanced from the IVC to the right hepatic vein and into the LA through the abnormal venous fistula. We exchanged the Judkins catheter for a $10 \mathrm{~F}$ Torqvue LP long sheath. An AMPLATZER $^{\mathrm{TM}}$ Vascular Plug II (AVP II) $(14 \mathrm{~mm} \times 10 \mathrm{~mm})$ was delivered successfully and occluded the Hepatic-LA fistula, with no obstruction across the Fontan tube (Figure 2). Arterial oxygen saturation increased to $100 \%$ with excellent hemodynamics.
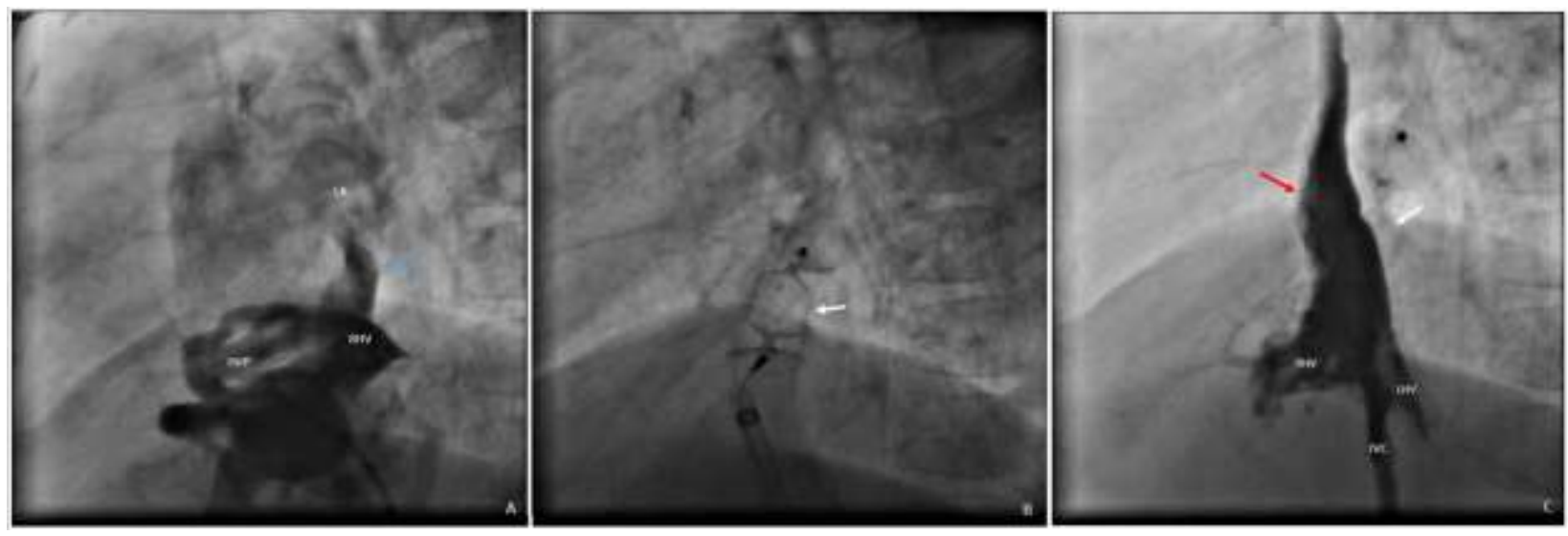

Figure 2: Angiograms in lateral projection $(A)$ right hepatic vein $(R V H)$, dividing into multiple sinusoidal channels in the liver $(H V P=$ hepatic venous plexus), and draining into the right sided pulmonary venous atrium (LA) through a fistula (blue arrow) (B) Amplatzer vascular plug type II (right arrow) placed in successfully in the fistula, attached to a delivery wire (C) Amplatzer vascular plug type II (white arrow) closing the fistula with no obstruction of the Fontan circulation (red arrow).

Three years following fistula's occlusion, the patient was doing well and

reporting good exercise tolerance. His arterial oxygen saturation was 
$99 \%$, and the previously noted fistula was completely occluded.

\section{Discussion}

The development of decompressing veno-venous collaterals is common following Fontan operations. This phenomenon is thought to be a biologic reaction to the increased systemic venous pressure changes associated with this type of circulation. Often, these vessels are of supradiaphragmatic origin [5].

The severe hypoxemia reaching an arterial oxygen saturation in the 70s encountered in our patient was due to an infradiaphragmatic right to left shunt draining the systemic venous circulation (Hepatic vein) into the heart (left atrium). Cardiac catheterization before the Fontan operation revealed a tiny hepatic venous to LA connection that progressed and markedly increased in size following the Fontan operation. Hepatic venous malformations are seen in $8 \%$ of heterotaxy patients undergoing Fontan procedures [9].The physiology of the Fontan operation might stimulate the enlargement of rudimentary embryologic connections from the systemic veins to the pulmonary veins or the pulmonary venous atrium, resulting in decompressing veno-venous collaterals and causing desaturation.

Any abnormal systemic venous to pulmonary venous circuits must be well studied before the Fontan and considered for closure if indicated as this connection may further progress and develop and lead to significant hypoxemia. Significant desaturation warrants diagnostic catheterization and trans-catheter occlusion of the collateral circulation if no contraindication is present. If the collateral circulation cannot be closed, patients should be treated as cyanotic heart disease patients and should be considered for anticoagulant medication.

Veno-venous collaterals-i.e. systemic venous vessels draining into pulmonary venous bed- are found in 20 to 45 percent of patients with Fontan circulation [3]. High-risk Fontan patients, such as patients with increased central venous pressures, may benefit from these collaterals; thus, collaterals closure may decrease survival rate [4]. Poterucha et al. Observed decreased 5-year survival in patients who had VVC embolization after Fontan [4]. However, his study included older patients with a mean age of 26 years and presented with mild arterial oxygen desaturation at $88-90 \%$ [5].

Since our patient had severe hypoxemia with arterial oxygen saturation reaching $70 \%$ and low mean pulmonary pressure of $14 \mathrm{~mm} \mathrm{Hg}$, we decided to close the fistula. The procedure was performed successfully without complication. The patient improved clinically immediately after closure of the veno-atrial fistula, with arterial oxygen saturation increased from $75 \%$ to $100 \%$ with a slight increase in the pulmonary arterial pressure from 14 to $16 \mathrm{~mm} \mathrm{Hg}$. A three year follow up revealed favorable results.

The relatively rare infradiaphragmatic VVCs, associated with the intrahepatic venovenous connection in the context of visceral heterotaxy presenting with severe cyanosis may be treated differently from those VVC in the older patients presenting with mild cyanosis. Reports of intrahepatic VVC presenting with profound cyanosis following the Fontan operation are rare and usually associated with visceral heterotaxy and a nest of intrahepatic venous plexus. The few reported cases that involved occlusion of these intrahepatic VVC were associated with a favorable outcome [5-10].

Veno-venous collaterals closure is still debatable since high-risk patients (patients with elevated central venous pressure or heterotaxies) might benefit from the natural fenestrations created by the veno-venous collaterals.

\section{Conclusion}

Closure of a fistula causing right to left shunt in patients post-Fontan procedure should be considered cautiously. Approach to venovenous collaterals in older patients after the Fontan operation may differ from that in younger children with severe hypoxemia and subdiaphragmatic intrahepatic VVC. Stimulation of the formation of intrahepatic venous plexus following the Fontan operation may cause opening or enlargement of accessory hepatic to atrium vessels, especially in patients with visceral heterotaxy, and lead to progressive cyanosis, despite an acceptable systemic venous pressure. Patients with significant cyanosis due to intrahepatic to atrial connection may benefit from the occlusion of these connections. The approach of the VVC arising from the hepatic veins maybe be different from the smaller VVC associated with mild desaturation. Percutaneous closure of hepatic to atrial connection in younger children with significant hypoxemia following appropriate hemodynamic assessment may be beneficial. We need to monitor longterm complications since there is not enough literature describing long term morbidity and mortality in similar patients.

Disclosure: The authors declare that the research was conducted in the absence of any commercial or financial relationships that could be construed as a potential conflict of interest.

\section{References}

1. Fontan F, Baudet E. Surgical repair of tricuspid atresia. Thorax 1971;26:240-248.

2. Rychik J, Atz AM, Celermajer DS, et al. Evaluation and Management of the Child and Adult with Fontan Circulation: A Scientific Statement From the American Heart Association. Circulation 2019. PMID: 31256636

3. Bouhout I, Ben-Ali W, Khalaf D, Raboisson MJ, Poirier N. Effect of Fenestration on Fontan Procedure Outcomes: A Meta-Analysis and Review. Ann Thorac Surg 2020.

4. Poterucha JT, Johnson JN, Taggart NW, et al. Embolization of Veno-venous Collaterals after the Fontan Operation Is Associated with Decreased Survival. Congenit Heart Dis 2015;10:E230-236.

5. Weber HS, Munir F, Stefek B, Lynch FC (2020). Percutaneous Amplatzer septal occluder device occlusion of a large portal vein to pulmonary venous atrium collateral resulting in severe systemic hypoxemia post Fontan. Congenital Cardiology today Vol. 18 - Issue 1, January, 2020.

6. Guzeltas A, Tanidir IC, MD, Saygi1 M. Major Intrahepatic Veno-Venous Fistula after Fontan Operation Treated by Transcatheter Implantation of Amplatzer Septal Occluder through Internal Jugular Vein. Braz J Cardiovasc Surg 2016;31(2):174-177

7. Guérin P, Losay J, Baron O. Transcatheter occlusion of an intrahepatic venovenous fistula after modified Fontan circulation by implantation of an Amplatzer atrial septal 
occluder. Catheter Cardiovasc Interv. 2005;64(1):117120.

8. Szkutnik M, Bialkowski J, Knapik P. Major intrahepatic veno-venous fistula after modified Fontan operation treated by transcatheter implantation of Amplatzer septal occluder. Cardiol Young. 2001;11(3):357-360.

9. Kuo JA. Percutaneous device occlusion of hepatocardiac venous collateral via left transhepatic access in a patient with heterotaxy syndrome following Fontan procedure. Catheter Cardiovasc Interv. 2015;85(5):E140-E143.

10. Rothman A, Acherman RJ, Luna CF, Restrepo H. Enlarged left vitelline vein remnant as a cause of cyanosis after the Fontan procedure: resolution with an Amplatzer vascular plug. Pediatr Cardiol. 2006;27(3):381-384.
This work is licensed under Creative Commons Attribution 4.0 License

\section{To Submit Your Article Click Here: Submit Manuscript}

DOI: $10.31579 / 2641-0419 / 148$
Ready to submit your research? Choose Auctores and benefit from:

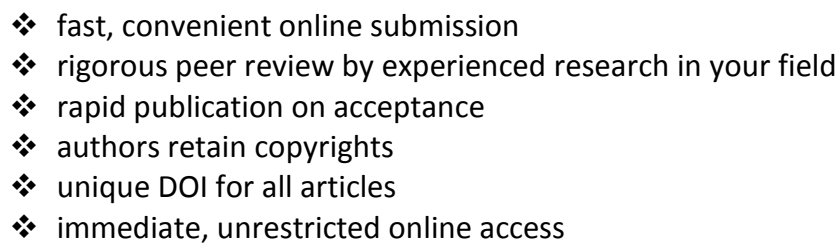

At Auctores, research is always in progress.

Learn more www.auctoresonline.org/journals/clinical-cardiology-andcardiovascular-interventions 